\title{
Evaluation of Tilt-correction of Anteversion on Anteroposterior Pelvic Radiographs in Total Hip Arthroplasty
}

\author{
Jeffrey M. Muir ${ }^{1}$, John Vincent ${ }^{2}$, Joseph Schipper ${ }^{3}$, Meinusha Govindarajan ${ }^{2}$, Wayne G. Paprosky ${ }^{4}$ \\ 1. Clinical Research, Intellijoint Surgical 2. Faculty of Applied Health Sciences, School of Public Health and Health \\ Systems, University of Waterloo 3. Research \& Development, Intellijoint Surgical 4. Surgery, Central Dupage Hospital
}

Corresponding author: Jeffrey M. Muir, j.muir@intellijointsurgical.com

\begin{abstract}
Despite inaccuracies due to artifact and variations in patient positioning, anteroposterior (AP) radiographs remain the clinical standard for post-operative evaluation of component placement following total hip arthroplasty (THA). However, cup position, specifically anteversion, can be significantly affected by variations in patient positioning on an X-ray. A major cause of such artifact is unaccounted for pelvic tilt. Several methods for correcting the effects of pelvic tilt on radiographic anteversion have been proposed, with varying degrees of accuracy. The purpose of this study was to evaluate the accuracy and reliability of a commonly referenced method for correcting acetabular cup anteversion in a cohort undergoing total hip arthroplasty and determine its appropriateness for use in this population of patients. Radiographs from patients who underwent primary or revision hip arthroplasty between February 2016 and February 2017 were retrospectively reviewed. Corrected anteversion was calculated by measuring the vertical distance between the symphysis pubis and the sacrococcygeal joint, per the method outlined by Tannast et al. This symphococcygeal distance was then applied to Tannast's nomograms to calculate the magnitude of pelvic tilt. Corrected and uncorrected anteversion values were compared to anteversion values collected intraoperatively using an imageless computer-assisted navigation device. A total of 71 cases were initially eligible for inclusion in the study. The correction method could not be applied in $44 \%$ (31/71) of the cases, chiefly due to difficulties in visualizing the required landmarks. In cases where it could be applied, corrected values correlated very poorly with navigation measurements $(r=-0.07)$. Mean corrected anteversion $\left(36.9^{\circ}\right.$, SD: $\left.7.4^{\circ}\right)$ differed from uncorrected anteversion $\left(25.2^{\circ}, \mathrm{SD}: 7.6^{\circ}\right)$ by an average of $13.5^{\circ}(\mathrm{p}<0.001)$. Mean navigated anteversion $\left(27.4^{\circ}, \mathrm{SD}: 5.7^{\circ}\right)$ differed from corrected values by an average of $10.8^{\circ}(\mathrm{p}=0.16)$. The evaluated correction method could not be consistently applied to radiographs and did not reliably correct anteversion due to pelvic tilt in this population of patients undergoing hip arthroplasty. This correction method does not appear to be appropriate for use in this patient population.
\end{abstract}

Received 04/18/2018

Review began 04/23/2018 Review ended 05/15/2018 Published 05/18/2018

๑) Copyright 2018

Muir et al. This is an open access article distributed under the terms of the Creative Commons Attribution License CC-BY 3.0., which permits unrestricted use, distribution, and reproduction in any medium, provided the original author and source are credited.
Categories: Orthopedics, Epidemiology/Public Health

Keywords: total hip arthroplasty, acetabular cup position, pelvic tilt, anteversion, tilt-correction

\section{Introduction}

Proper positioning of the acetabular cup component in total hip arthroplasty (THA) is vital; as inaccurate placement can lead to accelerated component wear, component loosening, reduced functional capacity, and an increased risk of impingement or dislocation [1-3]. Accurate radiographic assessment of cup orientation is therefore essential when evaluating the outcome of THA surgery.

Despite their known susceptibility to distortion and artifact, radiographs remain the standard of care for imaging, due to their low radiation exposure and easy accessibility [4]. Consistent positioning of patients during radiographs remains the chief challenge to imaging accuracy, as changes in leg adduction/abduction and pelvic tilt can dramatically alter the accuracy of measurements obtained from radiographs [5]. As such, plain radiographs may be inadequate for evaluating cup position if variations such as pelvic orientation are not taken into account [6].

Pelvic tilt has been a subject of much discussion regarding its effect on radiographic accuracy, as variations in pelvic tilt alter the projection of the pelvis onto the two-dimensional radiograph, introducing error to the measurement of acetabular orientation [7-8]. This error is generally considered negligible for inclination [9], but anteversion is highly susceptible to changes in pelvic tilt since its measurement is based solely on the shape of the ellipsis created by the cup surface on the radiograph [10]. To account for this projection error, several methods to correct pelvic tilt on radiographs have been presented in the literature [7,11], but have yet to achieve general acceptance for clinical use. In a recent study, Tannast et al. [7] evaluated several proposed measurements and found that the vertical distance from the pubic symphysis to the sacrococcygeal joint (symphococcygeal distance, $(\mathrm{SCD})$ ) correlated well with pelvic tilt. Using this method, the authors developed a nomogram for easy calculation of "tilt-corrected anteversion". However, the broad application of their preferred method may be difficult, as their radiographic methods were highly standardized and deviate from standard clinical procedure. Furthermore, as they used a cohort of young patients, the validity 


\section{Cureus}

of their correction method in an older population undergoing THA is in question.

The objective of this study was to evaluate the accuracy and reliability of the SCD method to correct radiographic anteversion on standard post-operative radiographs in a cohort of patients undergoing THA. We compared radiographic measurements before and after correction with values calculated from an imageless computer-assisted navigation system.

\section{Materials And Methods}

\section{Study design}

This investigation was a retrospective, single-centre, clinical study of patients undergoing primary or revision total hip arthroplasty. All included patients provided informed consent. Ethics approval was received prior to data collection.

\section{Patients}

Patients were eligible for inclusion if they underwent a primary or revision THA performed by the senior author (WGP) between February 2016 and February 2017. Specific inclusion criteria included THA using the Intellijoint $\mathrm{HIP}{ }^{\circledR}$ navigation system and ability to obtain two-week postoperative anteroposterior (AP) pelvic radiographs. Patients were excluded from analysis if the navigation tool was removed for any reason prior to the recording of necessary measurements, or if AP pelvic radiographs or navigation values were unable to be retrieved post-operatively.

\section{Radiographic data}

Radiographic analysis was performed on standing, two-week post-operative AP radiographs using TraumaCad (version 2.5, Brainlabs, Chicago, IL). Radiographs were scaled using the known diameter of the implanted femoral head. Pelvic tilt (deviation of the anterior pelvic plane (APP) from the coronal reference plane [11-12]) was estimated using the method described by Tannast et al. [7], where the vertical distance between the upper edge of the pubic symphysis to the centre of the sacro-coccygeal joint is measured. In this context, vertical was defined as a line perpendicular to the trans-ischial line, to account for any pelvic obliquity (Figure 1).

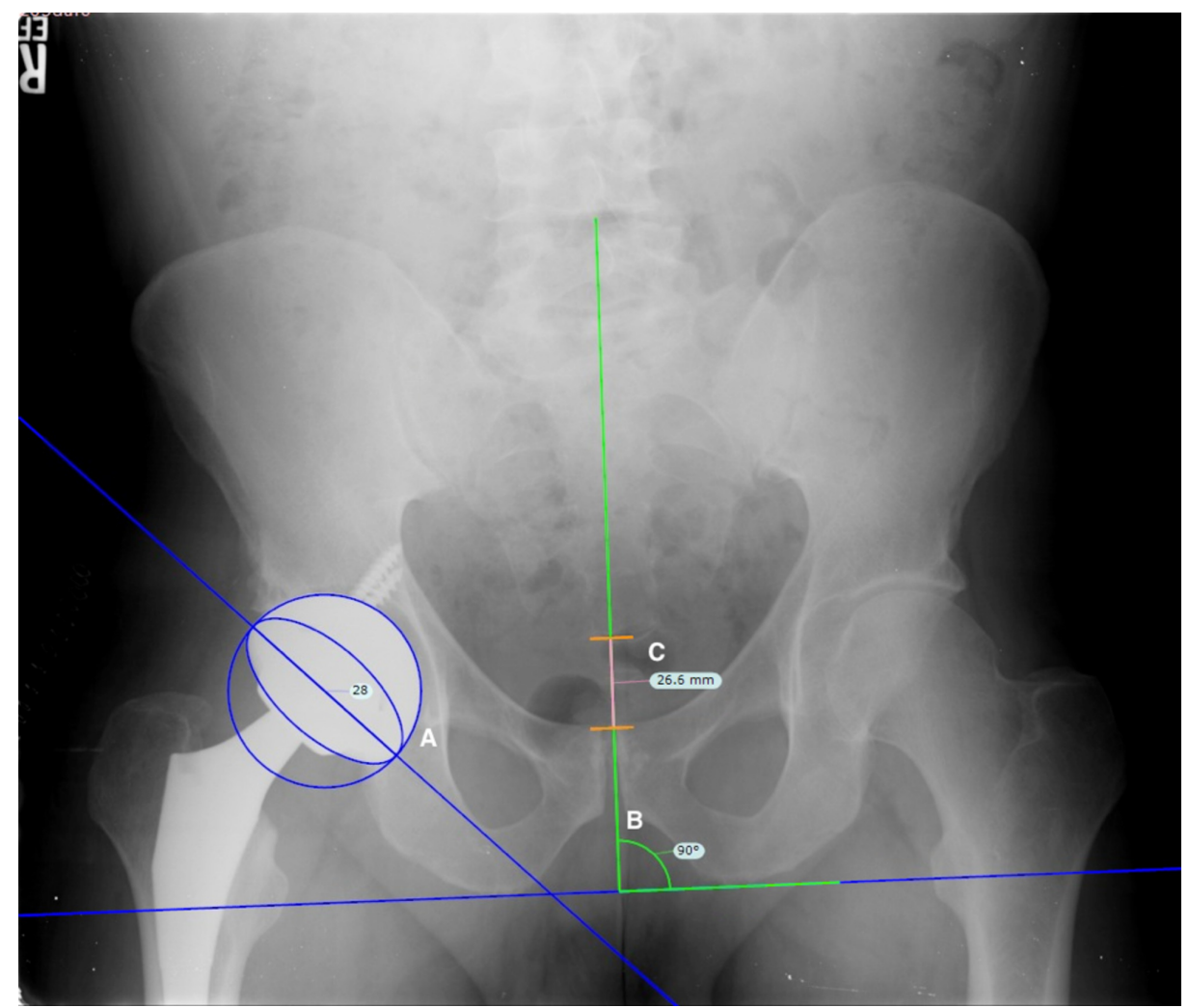

\section{FIGURE 1: Tannast method}

Radiographic analysis illustrating measured cup anteversion (A), the identification of "vertical" relative to the trans-ischial line $(B)$, and measured symphococcygeal distance from the pubic symphysis to the sacrococcygeal joint (C). 
Anteversion was measured using the trans-ischial line method. All radiographic measurements were made in triplicate and the results averaged to provide a final value. After completing radiographic measurements, the gender-specific nomograms created by Tannast et al. were used to find the tilt-corrected

anteversion. The nomograms correlate the SCD with the measured (uncorrected) anteversion to determine the tilt-corrected value. The measurements and application of the nomogram were completed by a single researcher (JV), who was blinded to navigation values.

\section{Navigation data}

Intraoperative measurements of anteversion were measured using a computer-assisted navigation device (Intellijoint HIP ${ }^{\circledR}$ navigation system, Intellijoint Surgical, Waterloo, ON). The indications and details on device use and workflow are described elsewhere [13-14]. In brief, the device consists of a camera, mounted to the operative iliac crest via two surgical pins, and a tracker, which can be magnetically fixed to a platform mounted to the greater trochanter or to surgical tools (e.g., impactor). During registration, patient position and pelvic orientation are registered relative to the patient's frontal plane. As the camera remains fixed to the patient during surgery and intraoperative measurements are calculated relative to this initial registration orientation, the resulting anteversion measurements represent the true in situ anteversion, not one distorted by imaging or intraoperative changes in pelvic position or orientation.

\section{Study outcomes and statistical analysis}

Acetabular cup anteversion measured from post-operative radiographs was compared with anteversion corrected using the nomograms provided by Tannast et al. [7] and anteversion measured intraoperatively by the navigation device.

Alpha was set a priori to $\mathrm{p}<0.05$ for all statistical comparisons between the radiographic and navigation data. Intra-observer reliability was assessed using the intra-class correlation coefficient. Mean values are expressed as mean (standard deviation) and compared using the mean differences and Student's t-test or single-factor ANOVA as appropriate. Correlations were evaluated using Pearson's r.

\section{Results}

\section{Patient population}

Seventy-seven eligible cases were completed during the study period. One patient was excluded due to the presence of a pelvic fracture on post-operative radiographs, and five patients were excluded due to extremely poor quality post-operative radiographs, from which accurate measurements were not possible. A final sample of 71 cases for which navigation data and uncorrected radiographs were available was included for analysis and comprised the baseline group.

In this baseline group, the sacrococcygeal joint was not visible in $44 \%$ (31/71) of radiographs. In the remaining 40 radiographs, the anteversion and/or SCD measurements calculated from the image were beyond the scale provided by the nomograms in $23 \%(9 / 40)$. As a result, the correction method was able to be successfully applied to only $44 \%$ (31/71) of radiographs from the initially eligible cases (correction-eligible group) (Figure 2). 


\section{Cureus}

\section{Initial Case Eligibility}

Cases initially eligible: 77

6 cases excluded:

- No post-op AP radiograph $(n=5$

- Visible pelvic fracture $(n=1)$

PSC Correction Eligibility

Cases eligible for inclusion: 71

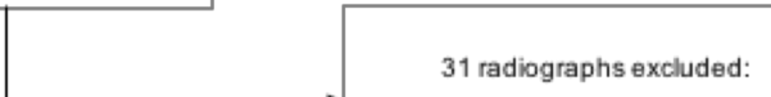

- PSC distance not measurable (coccyx not visible)

9 radiographs excluded:

- Values beyond nomogram boundaries

Cases where tilt-corrected

anteversion could be

calculated: 31

\section{FIGURE 2: Case flow-through}

Case flow-through and data collection eligibility.

No statistically significant differences were noted between gender distribution, operative side, or age in the baseline and correction-eligible groups (Table 1). 


\section{Cureus}

\begin{tabular}{|c|c|c|c|}
\hline & Baseline cohort $(n=71)$ & Correction-eligible cohort $(n=40)$ & p-value* \\
\hline Gender & & & $0.39^{\star \star}$ \\
\hline Male, $n(\%)$ & $38(54)$ & $18(45)$ & \\
\hline Female, n (\%) & $33(46)$ & $22(55)$ & \\
\hline Operative side & & & $0.84^{\star \star}$ \\
\hline Right, n (\%) & $27(38)$ & $16(40)$ & \\
\hline Left, $\mathrm{n}(\%)$ & 44 (62) & $24(60)$ & \\
\hline \multicolumn{4}{|l|}{ Age, mean (years) } \\
\hline Male and female & 63.3 & 63.1 & $0.88^{\star \star \star}$ \\
\hline Male only & 62.2 & 60.0 & $0.35^{\star \star \star}$ \\
\hline Female only & 64.7 & 65.8 & $0.59^{\star \star \star}$ \\
\hline
\end{tabular}

TABLE 1: Demographic statistics

\section{Intra-observer reliability}

Intra-observer reliability between the three rounds of measurement was excellent for both anteversion measured from radiographs (intraclass correlation $=0.99, \mathrm{SD}: 0.51^{\circ}$ ) and the symphococcygeal distance itself (intraclass correlation $=0.99$, SD: $0.25 \mathrm{~mm}$ ).

\section{Effect of correction on anteversion values}

Anteversion in the baseline and correction-eligible cohorts did not differ significantly for either radiographic or navigation measurements. Uncorrected radiographic measurements of anteversion were $26.3^{\circ}$ (SD: $6.8^{\circ}$ ) in the baseline cohort and $25.2^{\circ}$ (SD: $7.6^{\circ}$ ) in the correction-eligible cohort ( $\mathrm{p}=0.46$ ), while navigation values were $27.1^{\circ}\left(\mathrm{SD}: 5.3^{\circ}\right)$ and $27.4^{\circ}\left(\mathrm{SD}: 5.7^{\circ}\right)$, respectively $(\mathrm{p}=0.79)$.

In the correction-eligible cohort $(\mathrm{n}=40)$, uncorrected, corrected and navigation values differed significantly ( $F=27.38, \mathrm{p}<0.0001$ ). Corrected anteversion (mean: $36.9^{\circ}$, SD: $7.4^{\circ}$ ) differed from uncorrected anteversion (mean: $25.2^{\circ}$, SD: $7.6^{\circ}$ ) by an average of $13.5^{\circ}(\mathrm{p}<0.001)$ and from navigated anteversion $\left(27.4^{\circ}, \mathrm{SD}: 5.7^{\circ}\right)$ by an average of $10.8^{\circ}(\mathrm{p}=0.16)$. Navigation values did not differ significantly from uncorrected radiographic values $\left(27.4^{\circ}\left(\mathrm{SD}: 5.7^{\circ}\right)\right.$ vs. $\left.25.2^{\circ}\left(\mathrm{SD}: 7.6^{\circ}\right), \mathrm{p}=0.16\right)$. Corrected anteversion correlated very poorly with navigation values $(\mathrm{r}=-0.07)$ (Figure 3$)$ although moderately well with uncorrected values $(\mathrm{r}=0.73)$ (Figure 4). 


\section{Cureus}

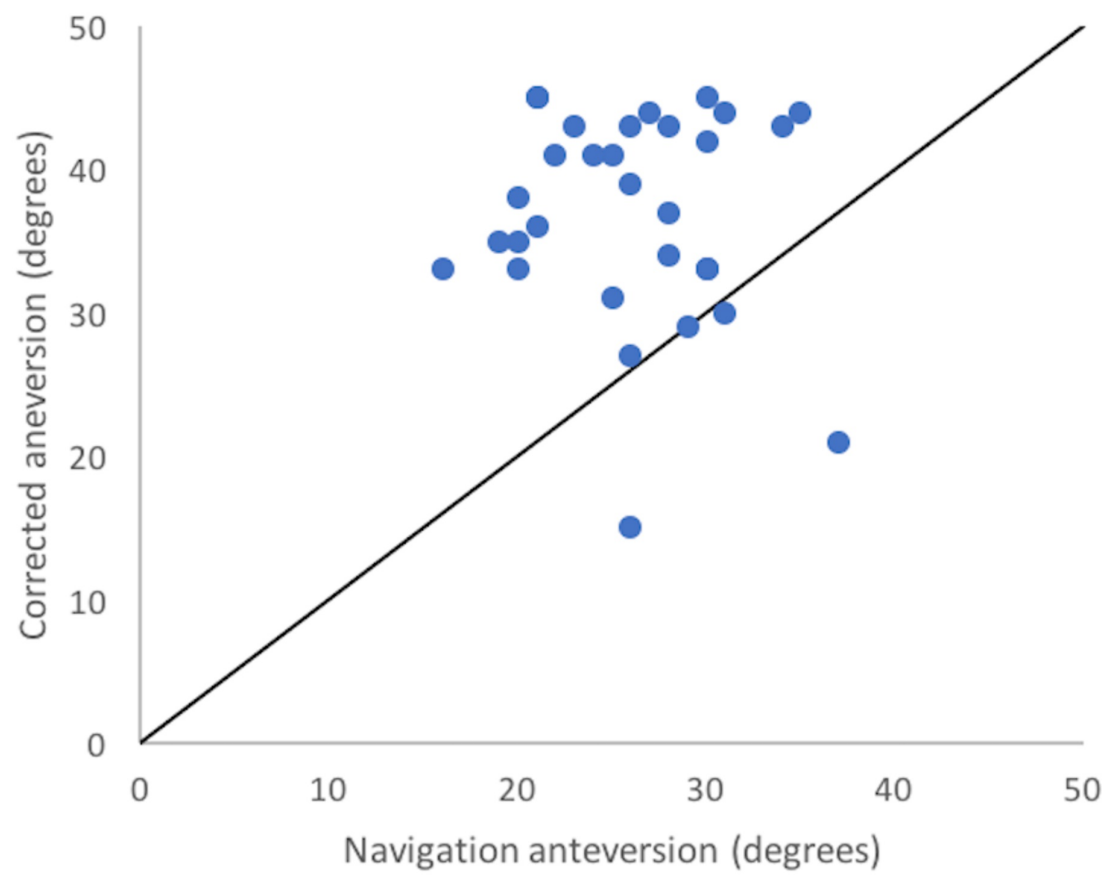

FIGURE 3: Corrected vs. navigated

Scatterplot showing the comparison between corrected anteversion values with navigation values.

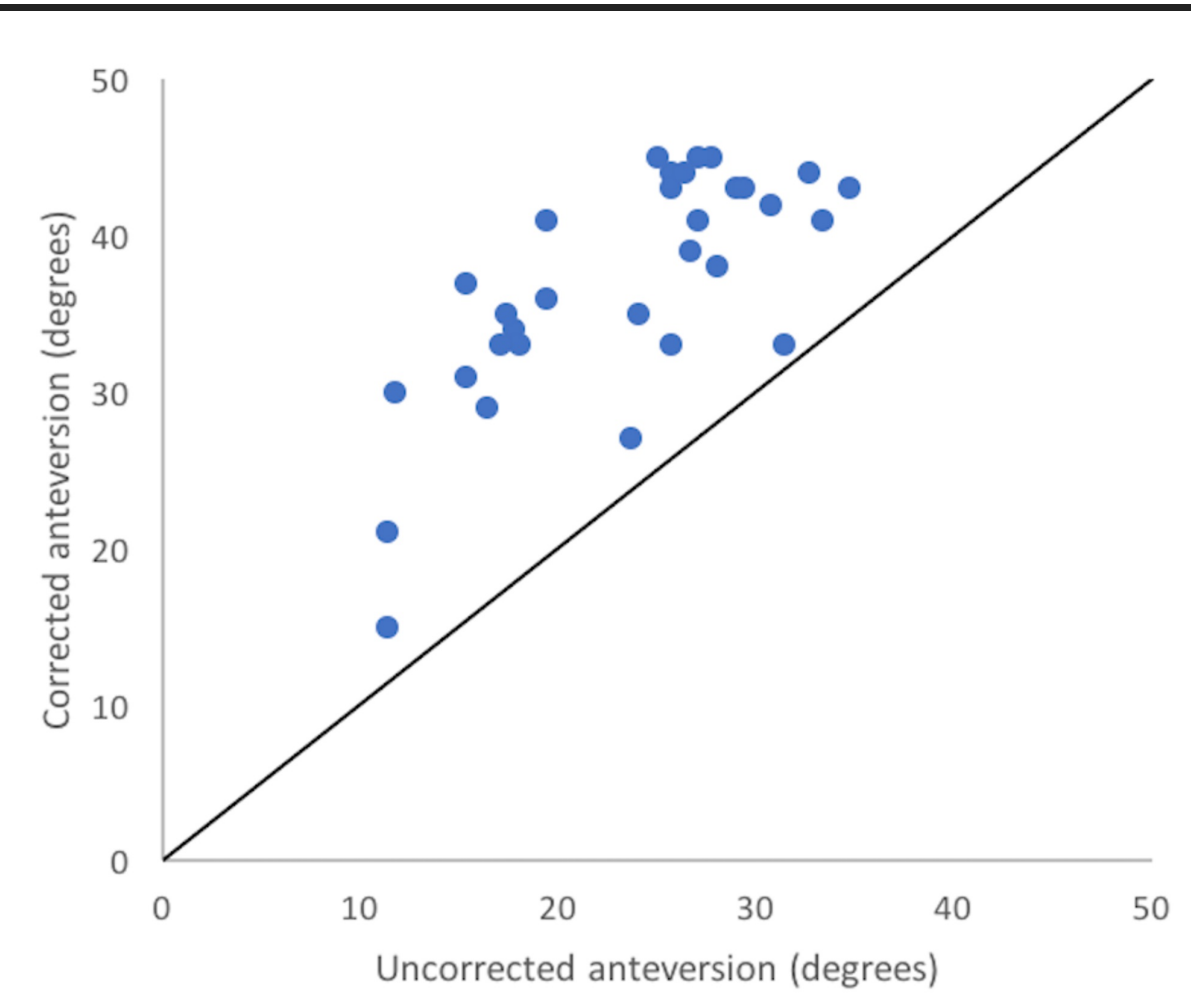

FIGURE 4: Corrected vs. uncorrected

Scatterplot showing the comparison between corrected anteversion values with uncorrected radiographic values.

Uncorrected anteversion correlated poorly with navigation values $(r=0.15)$ but differed by an average of 


\section{Cureus}

only $-2.2^{\circ}$ (SD: $8.7^{\circ}$ ) and demonstrated a more uniform distribution when compared with the uniform increase in anteversion values resulting from the correction method (Figure 5).

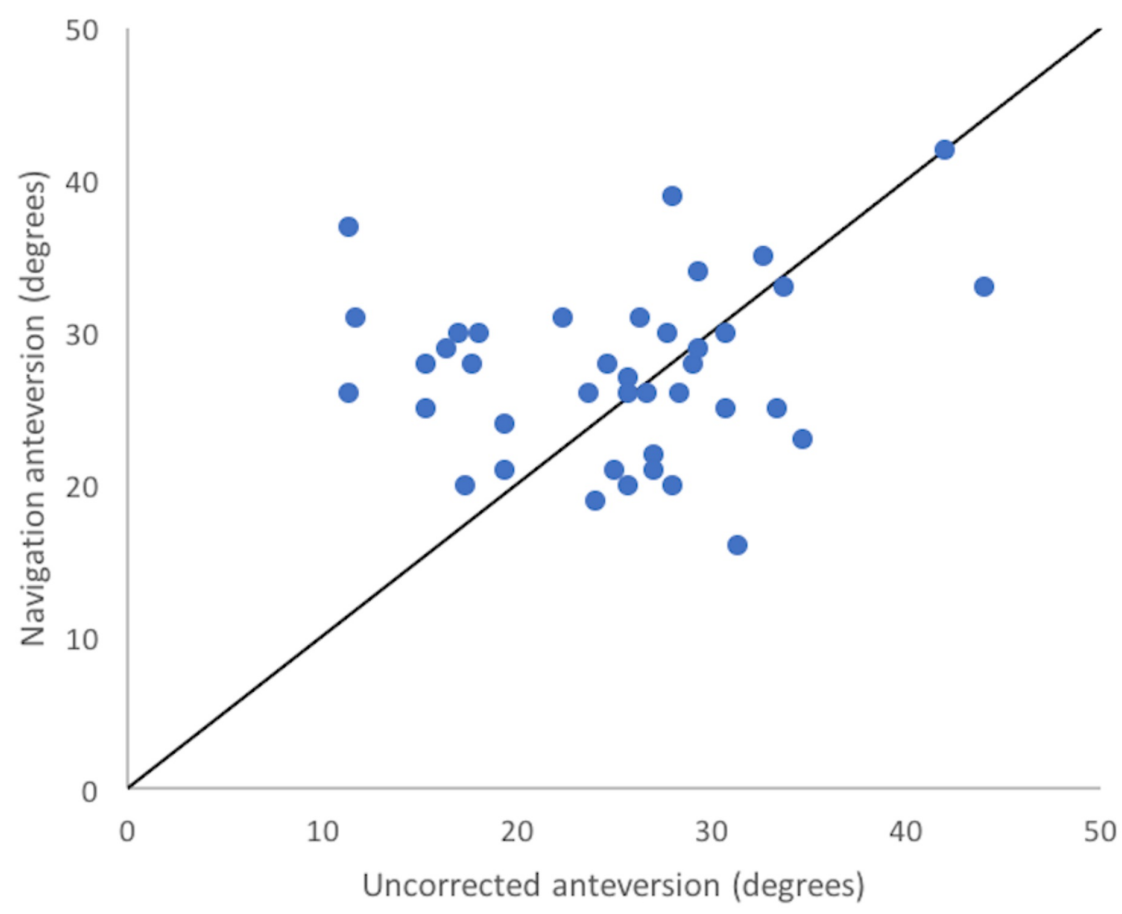

\section{FIGURE 5: Navigated vs. uncorrected}

Scatterplot illustrating the correlation between uncorrected and navigated anteversion values, showing uniform correlation between the two measurements.

\section{Discussion}

Accurate evaluation of acetabular cup position after THA is crucial for assessing treatment success, as malpositioning increases the risk of numerous post-operative sequelae that may necessitate further intervention $[9,15-16]$. While radiographs are the standard post-operative imaging modality, the inaccuracy of radiographic methods for determining cup anteversion and their inability to correct for positional errors such as pelvic tilt are well-documented [5-6,8,11,17]. Several methods for correcting for pelvic tilt have been suggested, with limited success. Our evaluation of one common correction method [7] found that this method did not provide a consistently valid correction for radiographic anteversion.

We noted several deficits when applying the SCD correction method that suggest that it may not provide a suitably reliable method for tilt correction. Primarily, the inability to reliably identify the required landmarks on radiographs presented a significant obstacle. We were unable to identify the coccyx in $44 \%$ of cases, immediately limiting our ability to broadly apply the correction method. In the group of cases where we were able to apply the correction, the measured values fell outside of the limits of the nomograms in a substantial portion of cases (23\%). Finally, in those cases where a corrected anteversion value could be calculated, there were significant differences between the corrected anteversion values and those calculated by the navigation device. No strong correlations were noted between corrected, uncorrected and navigation anteversion values. In fact, navigated values were more uniformly correlated with uncorrected values than corrected values. Corrected values were observed to be increased in all cases when compared with both navigated and uncorrected values. This trend towards increasing anteversion has important implications, as it could lead to overestimation of intraoperative anteversion, resulting in lower true anteversion orientations, a risk factor for posterior dislocation [18]. As such, careful interpretation of corrected anteversion is required to minimize the potential exposure of patients to increased risk of dislocation.

There were important methodological differences between our study and that of Tannast et al. that may help to explain the observed discrepancies. Firstly, Tannast et al. employed highly standardized radiographic techniques with a controlled source-to-image distance of $1.2 \mathrm{~m}$, patients in supine position, and the central beam aimed at the midpoint between the pubic symphysis and centre of the trans-ASIS line. This highly regimented approach is in contrast with standard post-operative AP radiographs, which are taken in either supine or standing position, with the central beam aimed at the pubic symphysis, and commonly with small 
variations in source-to-image distance [19]. Second, differences in population demographics (mean age: 31.7 yrs vs. $63.1 \mathrm{yrs}$ ) and indication (femora-acetabular impingement or developmental dysplasia vs. THA) and the associated anatomical differences that may account for the discrepancies and underscore the questionable validity of this correction method for THA patients. Finally, methods for measuring the SCD may be inconsistent between studies. Tannast et al. did not clarify whether their vertical distance was relative to a pelvic reference (such as the trans-ischial line) or relative to the plane of the X-ray, and failed to record if or how they scaled their radiographs. While most templating software assumes a magnification factor of $115 \%$ or $120 \%$ [20-21], true magnification can range from $97 \%$ to $127 \%$ [20-24]. We scaled radiographs using the known head size of the femoral implant, a reliable method that has been used elsewhere [21]. Since Tannast et al. used a highly standardized radiographic method, magnification should not impact their internal validity, but may explain the low compatibility of the correction method with the radiographs in the present study.

The retrospective nature of our study could be associated with limitations related to the consistency of the radiographic technique used, resulting in potential discrepancies between pre- and post-operative patient positioning. As well, comparing post-operative imaging with intraoperative measurements from navigation may represent a source of error, given that navigation measurements are intraoperative, while radiographs are post-operative. However, navigation in general [25-27] and the system utilized in this study specifically [13,28-29] have demonstrated excellent accuracy when compared with post-operative radiographs, with the Intellijoint system associated with an error of less than $3^{\circ}$ when compared with radiographs [29].

\section{Conclusions}

Our evaluation of a pelvic tilt correction method based on the symphococcygeal distance was associated with difficulties in identifying the required landmarks on X-ray and with significant discrepancies when compared with intraoperative measurements. While methodological differences may account for these discrepancies, the low reliability of the proposed method suggests it is not a viable option to correct for pelvic tilt in THA imaging. Further research is required to determine a more accurate method of tilt correction on radiographs.

\section{Additional Information \\ Disclosures}

Human subjects: Consent was obtained by all participants in this study. Veritas IRB issued approval 1621916:04:4816-02-2018. Unconditional approval granted on Feb 18, 2018. . Animal subjects: All authors have confirmed that this study did not involve animal subjects or tissue. Conflicts of interest: In compliance with the ICMJE uniform disclosure form, all authors declare the following: Payment/services info: All authors have declared that no financial support was received from any organization for the submitted work. Financial relationships: Muir, Schipper, Paprosky declare(s) personal fees, employment and stock/stock options from Intellijoint Surgical. JMM and JS are employees of and hold stock options in Intellijoint Surgical. WGP has received consultancy fees and holds stock options in Intellijoint Surgical. . Other relationships: All authors have declared that there are no other relationships or activities that could appear to have influenced the submitted work.

\section{References}

1. Beckmann J, Luring C, Tingart M, Anders S, Grifka J, Kock FX: Cup positioning in THA: current status and pitfalls. A systematic evaluation of the literature. Arch Orthop Trauma Surg. 2009, 129:863-872. 10.1007/s00402-008-0686-7

2. Sadhu A, Nam D, Coobs BR, Barrack TN, Nunley RM, Barrack RL: Acetabular component position and the risk of dislocation following primary and revision total hip arthroplasty: a matched cohort analysis. J Arthroplasty. 2017, 32:987-991. 10.1016/j.arth.2016.08.008

3. Sculco PK, McLawhorn AS, Carroll KM, McArthur BA, Mayman DJ: Anteroposterior radiographs are more accurate than cross-table lateral radiographs for acetabular anteversion assessment: a retrospective cohort study. HSS J. 2016, 12:32-38. 10.1007/s11420-015-9472-6

4. Shin WC, Lee SM, Lee KW, Cho HJ, Lee JS, Suh KT: The reliability and accuracy of measuring anteversion of the acetabular component on plain anteroposterior and lateral radiographs after total hip arthroplasty. Bone Joint J. 2015, 97:611-616. 10.1302/0301-620X.97B5.34735

5. Mellano CR, Spitzer AI: How does pelvic rotation or tilt affect radiographic measurement of acetabular component inclination angle during THA? J Orthop. 2015, 12:222-227. 10.1016/j.jor.2015.05.009

6. Craiovan B, Weber M, Worlicek M, et al.: Measuring acetabular cup orientation on antero-posterior radiographs of the hip after total hip arthroplasty with a vector arithmetic radiological method. Is it valid and verified for daily clinical practice?. Rofo. 2016, 188:574-581. 10.1055/s-0042-104205

7. Tannast M, Murphy SB, Langlotz F, Anderson SE, Siebenrock KA: Estimation of pelvic tilt on anteroposterior X-rays--a comparison of six parameters. Skeletal Radiol. 2006, 35:149-155. 10.1007/s00256-005-0050-8

8. Dandachli W, Ul Islam S, Richards R, Hall-Craggs M, Witt J: The influence of pelvic tilt on acetabular orientation and cover: a three-dimensional computerised tomography analysis. Hip Int. 2013, 23:87-92. 10.5301/HIP.2013.10715

9. Tiberi JV III, Antoci V, Malchau H, Rubash HE, Freiberg AA, Kwon YM: What is the fate of total hip arthroplasty (THA) acetabular component orientation when evaluated in the standing position? J Arthroplasty. 2015, 30:1555-1560. 10.1016/j.arth.2015.03.025 
10. Hassan DM, Johnston GH, Dust WN, Watson LG, Cassidy D: Radiographic calculation of anteversion in acetabular prostheses. J Arthroplasty. 1995, 10:369-372.

11. Kanazawa M, Nakashima Y, Arai T, et al.: Quantification of pelvic tilt and rotation by width/height ratio of obturator foramina on anteroposterior radiographs. Hip Int. 2016, 26:462-467. 10.5301/hipint.5000374

12. Maratt JD, Esposito CI, McLawhorn AS, Jerabek SA, Padgett DE, Mayman DJ: Pelvic tilt in patients undergoing total hip arthroplasty: when does it matter?. J Arthroplasty. 2015, 30:387-391. 10.1016/j.arth.2014.10.014

13. Grosso P, Snider M, Muir JM: A smart tool for intraoperative leg length targeting in total hip arthroplasty: a retrospective cohort study. Open Orthop J. 2016, 10:490-499. 10.2174/1874325001610010490

14. Paprosky WG, Muir JM: Intellijoint HIP: a 3D mini-optical navigation tool for improving intraoperative accuracy during total hip arthroplasty. Med Devices (Auckl). 2016, 9:401-408. 10.2147/MDER.S119161

15. Liaw CK, Yang RS, Hou SM, Wu TY, Fuh CS: Measurement of the acetabular cup anteversion on simulated radiographs. J Arthroplasty. 2009, 24:468-474. 10.1016/j.arth.2007.10.029

16. Moskal JT, Capps SG: Acetabular component positioning in total hip arthroplast: an evidence-based analysis. J Arthroplasty. 2011, 26:1432-1437. 10.1016/j.arth.2010.11.011

17. Bayraktar V, Weber M, von Kunow F, et al.: Accuracy of measuring acetabular cup position after total hip arthroplasty: comparison between a radiographic planning software and three-dimensional computed tomography. Int Orthop. 2017, 41:731-738. 10.1007/s00264-016-3240-1

18. Seagrave KG, Troelsen A, Malchau H, Husted H, Gromov K: Acetabular cup position and risk of dislocation in primary total hip arthroplasty: a systematic review of the literature. Acta Orthop. 2017, 88:10-17. 10.1080/17453674.2016.1251255

19. Derbyshire B: Correction of acetabular cup orientation measurements for X-ray beam offset . Med Eng Phys. 2008, 30:1119-1126. 10.1016/j.medengphy.2008.02.001

20. White SP, Bainbridge J, Smith EJ: Assessment of magnification of digital pelvic radiographs in total hip arthroplasty using templating software. Ann R Coll Surg Engl. 2008, 90:592-596. 10.1308/003588408X318101

21. Archibeck MJ, Cummins T, Tripuraneni KR, et al.: Inaccuracies in the use of magnification markers in digital hip radiographs. Clin Orthop Relat Res. 2016, 474:1812-1817. 10.1007/s11999-016-4704-8

22. Bayne CO, Krosin M, Barber TC: Evaluation of the accuracy and use of x-ray markers in digital templating for total hip arthroplasty. J Arthroplasty. 2009, 24:407-413. 10.1016/j.arth.2007.11.020

23. Descamps S, Livesey C, Learmonth ID: Determination of digitised radiograph magnification factors for preoperative templating in hip prosthesis surgery. Skeletal Radiol. 2010, 39:273-277. 10.1007/s00256-0090732-8

24. Heep H, Xu J, Lochteken C, Wedemeyer C: A simple and convenient method guide to determine the magnification of digital X-rays for preoperative planning in total hip arthroplasty. Orthop Rev (Pavia). 2012, 4:e12. 10.4081/or.2012.e12

25. Renkawitz T, Weber M, Springorum HR, et al.: Impingement-free range of movement, acetabular component cover and early clinical results comparing 'femur-first' navigation and 'conventional' minimally invasive total hip arthroplasty: a randomised controlled trial. Bone Joint J. 2015, 97:890-898. 10.1302/0301620X.97B7.34729

26. Lass R, Kubista B, Olischar B, Frantal S, Windhager R, Giurea A: Total hip arthroplasty using imageless computer-assisted hip navigation: a prospective randomized study. J Arthroplasty. 2014, 29:786-791. 10.1016/j.arth.2013.08.020

27. Gurgel HM, Croci AT, Cabrita HA, Vicente JR, Leonhardt MC, Rodrigues JC: Acetabular component positioning in total hip arthroplasty with and without a computer-assisted system: a prospective, randomized and controlled study. J Arthroplasty. 2014, 29:167-171. 10.1016/j.arth.2013.04.017

28. Paprosky WG, Muir JM: Improved accuracy of component placement during total hip arthroplasty: a retrospective cohort study. CCJR Winter Hip Course. Florida; 2017.

29. Vigdorchik JM, Cross MB, Bogner EA, Miller TT, Muir JM, Schwarzkopf R: A cadaver study to evaluate the accuracy of a new 3D mini-optical navigation tool for total hip arthroplasty. Surg Technol Int. 2017, 30:447454. 